\title{
LE MAITRE
}

DES

\section{HEURES DU IIARECIIL DE BOLCICAUT}

